Journal of Patient-Centered

\title{
Advancing Learning Health Systems Through Embedded Research: The 23rd Annual Conference of the Health Care Systems Research Network
}

\author{
Harold S. Luft \\ Ming Tai-Seale \\ Sarah M. Greene
}

Follow this and additional works at: https://aah.org/jpcrr

Part of the Clinical Epidemiology Commons, Community Health and Preventive Medicine Commons, Epidemiology Commons, Health Services Administration Commons, Health Services Research Commons, and the Public Health Education and Promotion Commons

\section{Recommended Citation}

Luft HS, Tai-Seale M, Greene SM, Advancing learning health systems through embedded research: the 23rd annual conference of the Health Care Systems Research Network. J Patient Cent Res Rev.

2017;4:139-43. doi: 10.17294/2330-0698.1460

Published quarterly by Midwest-based health system Advocate Aurora Health and indexed in PubMed Central, the Journal of Patient-Centered Research and Reviews (JPCRR) is an open access, peer-reviewed medical journal focused on disseminating scholarly works devoted to improving patient-centered care practices, health outcomes, and the patient experience. 


\title{
Advancing Learning Health Systems Through Embedded Research: The $23^{\text {rd }}$ Annual Conference of the Health Care Systems Research Network
}

\author{
Harold S. Luft, PhD, ${ }^{1}$ Ming Tai-Seale, PhD, MPH, ${ }^{2}$ Sarah M. Greene, $\mathrm{MPH}^{3}$ \\ ${ }^{1} \mathrm{Pal}$ Alto Medical Foundation Research Institute, Palo Alto, CA; Health Economics and Health Policy, University \\ of California, San Francisco, San Francisco, CA; ${ }^{2}$ Palo Alto Medical Foundation Research Institute, Palo Alto, CA; \\ Department of Health Research and Policy, Stanford School of Medicine, Stanford, CA; ${ }^{3}$ Health Care Systems \\ Research Network, Minneapolis, MN
}

\begin{abstract}
The 23rd annual conference of the Health Care Systems Research Network (HCSRN, formerly the HMO Research Network) was held in San Diego, California, March 21-23, 2017, attracting 387 attendees. As a consortium of 20 research organizations embedded in or affiliated with large health care delivery organizations, the HCSRN has held annual research conferences since 1994. The overall aim of the conferences is to bring researchers, project staff, research funders and other stakeholders together to share latest scientific findings and foster new research ideas and collaborations. The 2017 conference was hosted by the Palo Alto Medical Foundation Research Institute.

Each host site takes responsibility for the content and structure of the conference, and the 2017 team introduced several new features. In particular, past conferences used concurrent sessions to present research results in different topical areas, such as chronic disease, cancer, health informatics, mental health or precision medicine. This year, concurrent sessions shifted to panel discussions about how research results were achieved, including the use of methods, partnerships and analytic approaches. The 35 panels were organized into tracks such as engagement, data and informatics, partnerships and research implementation. Scientific results from HCSRN projects were presented via 120 posters in two poster sessions. Plenary sessions included a town hall-style panel with different funding agency representatives, an opening presentation on the range of opportunities and benefits to studying health systems, and a concluding presentation on how researchers can apply design thinking in their work. (J Patient Cent Res Rev. 2017;4:139-143.)
\end{abstract}

Keywords learning health system, health services research, health care

$\mathrm{R}$ eal-world health care delivery systems provide unique advantages for conducting research and populations. Assets these systems offer include: automated data from electronic health records (EHRs)

Correspondence: Sarah M. Greene, MPH,

Health Care Systems Research Network, c/o HealthPartners Institute, Mailstop 23301A, PO Box 1524, Minneapolis, MN, 55440, T: +1-206-819-3579,

Email: Sarah.M.Greene@HealthPartners.com and health care claims; a stable population base that can be observed across time and care settings; and the ability to study the impact of myriad changes in how care is organized, financed and delivered.

The Health Care Systems Research Network (HCSRN) was conceived in 1994 by leaders from six research centers embedded in delivery systems, including Kaiser Permanente and others. Since then, HCSRN has grown to include 20 health systems with ability to conduct multisite clinical trials, epidemiologic studies and comparative studies of health care services. HCSRN's 
mission - to improve individual and population health through research that connects the resources and capabilities of learning health care systems - conveys both a commitment to public domain research and the importance of connectivity. The connections and culture of collaboration assist HCSRN in competing for research funding from federal and other sources, and contribute to its ability to inform and influence care.

A common data model is the backbone of the research network, allowing data to be organized in a virtual data warehouse (VDW). It is virtual in that local health system data remain in place at a local data warehouse, using standardized processes for improving data quality and validity as well as governance of data sharing. Each HCSRN site incorporates information from EHRs and other clinical and administrative sources into a set of VDW data tables with standardized variable names, labels, definitions and coding. The data analysts and IT staff who develop and support this VDW are critical to the network's success, and notably, they maintain regular connectivity and collaborative structures that mirror those of HCSRN scientists.

Each year since its inception, the HCSRN has held an annual conference to showcase its scientific findings ${ }^{\text {cf.1, } 1,2}$ and to incubate new collaborations. The conference is a centerpiece of HCSRN's collaborative infrastructure. The 2017 conference - which followed the theme "Advancing learning health systems through embedded research" - was hosted by the Palo Alto Medical Foundation Research Institute. Each host site takes responsibility for the content and structure of the conference, and the 2017 team introduced several new features described herein.

\section{New Approach to Conference Programming}

In planning the 2017 meeting, the conference committee decided to shift emphasis to focus on the uniqueness of HCSRN and the ways its researchers do their work. The organizers sought to make this meeting different from other professional meetings - one worth going to in this environment of uncertainty and tight budgets.

HCSRN researchers typically belong to other professional associations, ranging from health economics to medical anthropology to AcademyHealth, and attend many subject-oriented meetings such as the
Society for Medical Decision Making and American Society of Preventive Oncology. The commonality throughout HCSRN is that it is an organization of "embedded researchers" - people conducting health science research through positions inside health care systems. There also are embedded researchers in organizations outside the network, such as the Department of Veterans Affairs, who may benefit from the content of the HCSRN conference, and the planners crafted the conference program with the hope it also would attract non-HCSRN researchers.

Notably, researchers in academic settings often work adjacent to medical centers, but typically do not have access to the detailed data and insights of the "operational" parts of those systems. Moreover, they often follow the usual research model of beginning with a question that is theoretically interesting (ie, filling a gap in scientific knowledge) and then seeking data to test the resulting hypotheses. Embedded researchers sometimes operate in this fashion. Being inside the delivery system, however, they also can discover problems facing front-line clinicians and operational leaders. They often have the skills to address those problems in a rigorous fashion and, in doing so, may uncover generalizable lessons to advance science and care delivery elsewhere.

This additional pathway to doing public domain research has both advantages and disadvantages. Among the advantages is the pursuit of research intended to be practical — that which directly addresses problems that arise in real-world health care and offers a relevant solution to accompany peerreviewed publications that are the bulwark of scientific research. Another advantage, increasingly important in an uncertain funding climate, is that the parent health care delivery systems in which HCSRN researchers work may be willing to fund some studies if they are deemed pertinent and of high priority.

Leveraging these opportunities, however, requires approaches to research that may differ from approaches used to successfully compete for federal funding. Investigator-initiated research proposals seeking federal support from the National Institutes of Health often take months of preparing tightly structured hypothesis tests and reference-dense literature reviews 
to meet the expectations of peer reviewers, then face a low probability of funding based on agency budgets relative to the number of well-scored/high-quality proposals. In contrast, internal funding often confers a "go/no-go" decision within weeks or months, but may carry the expectation of delivering meaningful findings within a year or so. The approach one uses to present both the project design and the findings for internally funded work may differ significantly from that used in the usual research setting.

Successful embedded researchers have a fluency in several techniques: forging strong relationships with operational leaders and clinicians; presenting compelling cases for internal funding; sharing actionable findings in a simple, clear fashion; and then presenting their work in a manner passing peer review in high-quality journals.

Thus, the planning team decided to use the 2017 HCSRN conference to share lessons on how to be better embedded researchers, and crafted the conference theme accordingly. In addition, the planners made several structural changes to the conference to facilitate this frameshift. The biggest change was a decision to reserve all the concurrent sessions for panels focusing on the "how" of doing embedded research, and sharing all scientific findings via poster presentations. This tactic also allowed for deeper engagement with poster authors, arguably a more effective way to learn about each author's research and its implications.

The call for abstracts for panel presentations was configured to address a range of topics designed to appeal to a broad swath of attendees, such as presentations on effective partnerships between researchers and delivery systems, overcoming challenges of integrating studies into health care operations, and informatics approaches that utilize the comprehensive data resources available in HCSRN systems. The response to this new format was reassuring, especially given the marked changes involved and the need to formulate panels among individuals who had not previously collaborated. Altogether, 35 panels were organized into tracks (patient and stakeholder engagement, data and informatics, learning health systems, partnerships) and presented over the course of the conference.
The call for abstracts for scientific results also was successful, garnering 120 accepted submissions. These abstracts are published within this issue of the Journal of Patient-Centered Research and Reviews, allowing HCSRN science to be shared with a wider audience. ${ }^{3}$

\section{The Plenary Sessions}

Richard Kronick, a professor at the University of California at San Diego and former director of the U.S. Agency for Healthcare Research Quality (AHRQ), was the plenary speaker for the opening session. Entitled "A Research Agenda on Comparative Health System Performance," his presentation outlined progress and remaining work to be done related to health care access, cost and quality.

Dr. Kronick delineated a research agenda on health system performance, describing the instrumental need to study health care to figure out how best to harvest and spread improvement. He noted opportunities for which HCSRN researchers may excel: develop and disseminate information on what works and what does not; address health planning/workforce issues; sharpen focus on disparities in outcomes for vulnerable populations within the health system and test methods of reducing those disparities; and use EHR information to develop measures of outcomes that matter to patients, clinicians and system managers. Robust audience discussion with Dr. Kronick occurred after the presentation.

Representatives from three funding agencies - Ann Geiger from the National Cancer Institute (NCI), Steven Clauser from the Patient-Centered Outcomes Research Institute (PCORI), and Sharon Arnold from AHRQ - comprised a town hall panel for the second day's plenary session. Each panelist offered opening remarks on the priorities of his/her agency, particularly as they related to population health and delivery system science.

Among the highlights, Dr. Arnold discussed anticipated funding opportunities from AHRQ for training researchers in how to conduct research in health systems, Dr. Clauser described PCORI's commitment to reducing disparities in the delivery of health care services, and Dr. Geiger referred the group to NCI's newly established program in cancer care delivery research. ${ }^{4}$ The timing of the conference, amid urgent national discussions about health care reform 
and the federal budget, led to interesting dialogue as researchers expressed their uncertainty about federal funding for research during a question-and-answer segment moderated by HCSRN Executive Director Sarah Greene.

Another plenary session, delivered annually at this conference, focused on the state of HCSRN. Stephen Waring, chair of the network's governing board, delivered opening remarks on how to leverage the historic successes of the HCSRN in support of new and pioneering work, pointing to the federal Precision Medicine Initiative as one example.

Ms. Greene followed with a presentation about how to ensure that HCSRN delivers value to its many constituencies - researchers, parent health systems and clinicians, patients and their families, policymakers, and the general public. Reflecting on a recent article on the value of biomedical research, which stated, "The criteria by which scientists and their teams are rewarded for their efforts by agencies that fund them and institutions that host them should align criteria with the desired outcomes: research that is productive, high-quality, reproducible, shareable, and translatable - or PQRST for short,"5 Ms. Greene observed that these are attributes already present in much of HCSRN's research and that participant-centered is another "P-word" that applies to the network.

The closing plenary session was given by Chris Waugh, chief innovation officer of Sutter Health (San Francisco, CA). The audience appreciated his inspiring presentation on applying human-centered design to guide Sutter Health's innovation strategy, develop and manage its innovation portfolio and enhance its reputation as a market leader. Drawing from more than a decade of entrepreneurial leadership experience at multiple Bay Area firms, he encouraged participants to learn from human-centered approaches to delight patients, remove barriers in health care providers' daily practice and create a culture of innovation. These approaches could be applied in various aspects of research, from selecting a study question to design and methodology.

\section{Additional Conference Highlights}

Along with the aforementioned panels that focused on aspects of how certain research was implemented, how stakeholder engagement affected a research project and how meaningful engagement with health system stakeholders was conducted, there were a number of other special sessions.

A panel on mentoring and training drew participants from a broad range of organizations and involved discussions on the characteristics of effective mentors, the importance of a "palette" of mentors rather than a single mentor, and how to give and get mentoring at different career stages. The audience, primarily early career faculty and project management staff, exhorted HCSRN to invest in mentoring scientists and encourage interorganizational mentoring.

Another unique and highly interactive session taught participants how to use techniques from the arts (storytelling, music, visual arts) to support more effective dissemination of research results.

A vital part of the conference was the convening of scientific interest group meetings and similar ancillary meetings at which researchers assembled to discuss specific new projects, review progress on existing projects or share insights and best practices in specific domains (eg, patient engagement or data science). This year, 33 groups convened formally, with several impromptu meetings deriving from new connections and shared interests. It was notable that 20 new organizations were in attendance this year and more than one-third of attendees were joining for the first time.

As it has matured and grown, HCSRN has expanded awards and honors bestowed at the conference. This year's conference presented awards for outstanding research paper, early career investigator, best poster from each of the two sessions and mentor of the year. Recipients were:

- Early Career Investigator: Jing Hao, Geisinger Health System (Danville, PA);

- HCSRN Paper of the Year: Cabell Jonas, Kaiser Permanente Mid-Atlantic States (Rockville, MD);

- Best Poster (Session 1): Lisa Carter-Harris, Indiana University (Bloomington, IN);

- Best Poster (Session 2): Ketan Mane, Kaiser Permanente Mid-Atlantic States;

- HCSRN Mentor of the Year: Harold (Hal) Luft, Palo Alto Medical Foundation Research Institute (Mountain View, CA). 


\section{HCSRN Annual Conference}

Planning is underway for the 2018 HCSRN conference, which will be held April 11-13 in Minneapolis, Minnesota. Details regarding abstract submission are available at www.hcsrnmeeting.org. On that site, as well as HCSRN's main web page, www.hcsrn.org, additional information is provided about the network's capabilities, public domain resources and scientific accomplishments.

\section{Acknowledgments}

The authors thank the 2017 HCSRN planning committee, the conference management team at Conference Solutions, Inc (Portland, OR) and the HCSRN governing board for their contributions and support. We gratefully acknowledge financial sponsorship from Sutter Health (Sacramento, CA) and the Patient-Centered Outcomes Research Institute (Washington, DC). Finally, we thank the faculty and staff from Sutter Health's Palo Alto Medical Foundation Research Institute as well as Research,
Development and Dissemination department for serving as volunteers at the conference, which would not have been possible without their dedication.

\section{References}

1. Abstracts from the 21st annual HMO Research Network conference, March 11-13, 2015, Long Beach, California. J Patient Cent Res Rev. 2015;2(S1):S78-141. CrossRef

2. Abstracts from the 22nd annual Health Care Systems Research Network Conference, April 13-16, 2016, Atlanta, Georgia. J Patient Cent Res Rev. 2016;3:167-228. CrossRef

3. Abstracts from the 23rd annual Health Care Systems Research Network Conference, March 20-23, 2017, San Diego, California. J Patient Cent Res Rev. 2017;4:144-202.

4. Geiger AM, Smith AW, Kobrin SC, Taplin SH. Enhancing healthcare delivery research at the National Cancer Institute. Am J Manag Care. 2016;22:SP502, 553-5. http:/www.ajmc. com/journals/evidence-based-oncology/2016/october-2016/ enhancing-healthcare-delivery-research-at-the-nationalcancer-institute/p-1. October 17, 2016. Accessed June 2, 2017.

5. Ioannidis JP, Khoury MJ. Assessing value in biomedical research: the PQRST of appraisal and reward. JAMA. 2014;312:483-4. CrossRef

(C) 2017 Aurora Health Care, Inc. 